\title{
Toxicidade de espiromesifeno e acaricidas naturais para Tetranychus urticae koch e compatibilidade com Phytoseiulus macropilis (Banks)
}

\author{
Toxicity of spiromesifen and natural acaricides to Tetranychus \\ urticae koch and compatibility with Phytoseiulus macropilis (Banks)
}

\author{
Alberto Belo Esteves Filho ${ }^{1 *}$; José Vargas de Oliveira ${ }^{2}$; Jorge Braz Torres²; \\ Cláudia Helena Cysneiros Matos ${ }^{3}$
}

Resumo

O ácaro rajado, Tetranychus urticae Koch (Acari: Tetranychidae) é uma importante praga do algodoeiro por ocasionar perdas na produção e na qualidade da fibra. Assim, este trabalho se propôs a avaliar a toxicidade do acaricida sintético espiromesifeno e produtos naturais para T. urticae e a compatibilidade com o seu predador Phytoseiulus macropilis (Banks) (Acari: Phytoseiidae). Espiromesifeno e os naturais Azadiractina A/B, Azadiractina 1\%, Jatropha curcas L. e Ricinus communis L. foram utilizados em diferentes concentrações; usou-se a técnica de imersão de discos de folha de algodão nas caldas dos produtos. As mortalidades de fêmeas e ovos de T. urticae foram avaliadas após 48 e $96 \mathrm{~h}$ da aplicação, respectivamente, visando à obtenção das concentrações letais. Estudou-se o efeito sobre P. macropilis, com base nas $\mathrm{CL}_{50 \mathrm{~s}}$ obtidas para T. urticae. Todos os acaricidas testados foram eficientes no controle de fêmeas e ovos de T. urticae. Entretanto, de acordo com as $\mathrm{CL}_{50 \mathrm{~s}}$ e $\mathrm{CL}_{90 \mathrm{~s}}$, espiromesifeno foi o mais tóxico para fêmeas e o óleo de $J$. curcas apresentou a maior toxicidade para ovos. Espiromesifeno, J. curcas e Azadiractina $1 \%$ causaram efeito adverso para P. macropilis. No entanto, espiromesifeno foi o único classificado como nocivo para o predador, enquanto Azadiractina $\mathrm{A} / \mathrm{B}$ e $R$. communis foram levemente nocivos. R. communis e Azadiractina $\mathrm{A} / \mathrm{B}$ foram eficientes no controle do ácaro rajado e promissores para o manejo desta praga no agroecossistema algodoeiro, considerando sua baixa toxicidade para o predador. Palavras-chave: Acari, algodão, controle químico, controle alternativo, controle biológico

\footnotetext{
Abstract

The two-spotted spider mite, Tetranychus urticae Koch (Acari: Tetranychidae), is one important pest of cotton crop due to reductions in cotton yield and fiber quality. Thus, this work evaluated the toxicity of the synthetic acaricide spiromesifen and natural products on $T$. urticae and the compatibility with the predatory mite Phytoseiulus macropilis (Banks) (Acari: Phytoseiidae). Spiromesifen and the natural products Azadirachtin A/B, Azadirachtin 1\%, Jatropha curcas L. and Ricinus communis L. oils were used at different concentrations; the leaf dipping method was employed. The mortality of T. urticae females and eggs was evaluated 48 and $96 \mathrm{~h}$ after treatment to calculate the lethal concentrations. The effect on P. macropilis was studied using the $\mathrm{LC}_{50 \mathrm{~s}}$ obtained to T. urticae. All acaricides tested were effective in controlling females and eggs of T. urticae. However, according to the $\mathrm{LC}_{50 \mathrm{~s}}$ and $\mathrm{LC}_{90 \mathrm{~s}}$

${ }^{1}$ Discente do Programa de Pós-Graduação em Entomologia Agrícola, Universidade Federal Rural de Pernambuco, UFRPE, Recife, PE. E-mail: betobelo@gmail.com

${ }^{2}$ Profs. do Programa de Pós-Graduação em Entomologia Agrícola, UFRPE, Recife, PE. E-mail: vargasoliveira@uol.com.br; jtorres@depa.ufrpe.br

${ }^{3}$ Prof ${ }^{\mathrm{a}}$ da Unidade Acadêmica de Serra Talhada, UAST, Serra Talhada, PE. E-mail: ccysne@hotmail.com

* Autor para correspondência
} 
calculated, spiromesifen was the most toxic acaricide to females and $J$. curcas oil presented the higher toxicity to eggs. Spiromesifen, J. curcas oil and Azadiractina 1\% caused side effects on P. macropilis. However, only espiromesifeno was classified as harmful to the predator, whereas Azadirachtin A/B and $R$. communis oil were slightly harmful. $R$. communis and Azadirachtin $\mathrm{A} / \mathrm{B}$ were effective in controlling the two-spotted spider mite and promising for the management of this pest in cotton considering their low toxicity to the predator.

Key words: Acari, cotton, chemical control, alternative control, biological control

\section{Introdução}

A compatibilidade de acaricidas e ácaros predadores é relevante para diminuir a frequência da aplicação de acaricidas para o manejo do ácaro rajado Tetranychus urticae Koch (Acari: Tetranychidae) (POLETTI; COLLETTE; OMOTO, 2008). Os inimigos naturais são responsáveis pela manutenção do equilíbrio populacional de um grande número de pragas no agroecossistema do algodão com apenas algumas espécies atingindo o status de praga (DEGRANDE et al., 2002; TORRES, 2008). Neste sentido é fundamental conhecer a compatibilidade de acaricidas sintéticos e naturais com os ácaros predadores fitoseídeos (MCMURTRY; CROFT, 1997), pois ambos são eficazes no manejo do ácaro rajado, entretanto, é possível que estes acaricidas possam causar efeitos adversos sobre as populações destes inimigos naturais (OVERMEER; VAN ZON, 1982).

O ácaro rajado T. urticae destaca-se como praga de grande impacto em diversas culturas agrícolas. A ocorrência desta espécie alimentando-se de plantas é registrada para mais de 1.100 espécies, pertencentes a 140 famílias botânicas, incluindo frutíferas, olerícolas e ornamentais (GRBIC et al., 2011). No algodoeiro, o ácaro rajado causa injúrias acentuadas nas folhas acarretando a queda precoce e consequente redução da produtividade e da qualidade das fibras (OLIVEIRA; CALCAGNOLO, 1975).

O controle de T. urticae em algodoeiro no Brasil é feito normalmente através de pulverizações com acaricidas, sendo registrados no país 48 produtos comerciais para o seu controle (AGROFIT, 2011). Acaricidas sintéticos de classes distintas têm apresentado eficiência no controle desta praga (ASHLEY et al., 2006; MARCIC, 2007; ESTEVES FILHO; OLIVEIRA; GONDIN JUNIOR, 2008). Entretanto, o manejo inadequado desses produtos tem proporcionado a seleção de populações resistentes, além dos fenômenos de ressurgência e surtos de pragas secundárias (SATO et al., 2005, 2007).

Dentro do manejo de T. urticae em diferentes culturas, óleos essenciais e extratos de plantas têm sido avaliados com sucesso no controle de formas imaturas e adultas desta praga (MIRESMAILLI; ISMAN, 2006; PONTES et al., 2011). Dentre as plantas como fontes de inseticidas naturais, o nim (Azadirachta indica A. Juss) tem se destacado pela sua eficiência por afetar negativamente a biologia e o comportamento de pragas, apresentar baixa toxicidade para vertebrados, além da seletividade a diversos inimigos naturais (CASTAGNOLI et al., 2002; MOURÃO et al., 2004; MARTINEZVILLAR et al., 2005).

Oácaropredador Phytoseiulusmacropilis(Banks) tem sido encontrado, frequentemente, nos estudos sobre a diversidade de ácaros no Brasil (REIS et al., 2000; VASCONCELOS et al., 2006). Em pepino e morangueiro (WATANABE et al., 1994; FERLA; MARCHETTI; GONÇALVES, 2007) observou-se a sua predação sobre $T$. urticae; também apresenta potencial para ser usado no controle biológico desta praga em algodoeiro (ESTEVES FILHO et al., 2010). Fato este justificado pelo hábito alimentar deste predador que, de acordo com Mcmurtry e Croft (1997) é especializado na predação de ácaros do gênero Tetranychus. Predadores especialistas se alimentam preferencialmente da praga-alvo, 
reduzindo rapidamente sua população (HOY, 2011). Em morangueiro, este predador é capaz de extinguir uma colônia de T. urticae em 20 dias na proporção de 1 predador para 100 fitófagos (OLIVEIRA et al., 2009). Adicionalmente, a quantidade de ovos produzidos por fêmea de $P$. macropilis é diretamente proporcional ao número de T. urticae consumidos (FERLA et al., 2011).

Assim, o presente trabalho teve os objetivos de avaliar a toxicidade de acaricidas sintéticos e produtos naturais sobre fêmeas e ovos de T. urticae, bem como determinar a compatibilidade dos acaricidas com o predador P. macropilis.

\section{Material e Métodos}

Os experimentos foram conduzidos no Laboratório de Entomologia Agrícola da Área de Fitossanidade da Universidade Federal Rural de Pernambuco (UFRPE), a partir de ácaros obtidos de criações já estabelecidas no laboratório.

Criação de Tetranychus urticae. Os ácaros foram criados por várias gerações em plantas de feijão de porco (Canavalia ensiformis D.C.), cultivadas em vasos plásticos de 5L de capacidade, contendo solo arenoso e mantidas em casas-de-vegetação. Após 10 dias do plantio, as plantas foram infestadas com fêmeas adultas do ácaro rajado, retiradas da criaçãoestoque do laboratório. A infestação foi efetuada com folhas de plantas já infestadas colocadas sobre as plantas sadias.

\section{Obtenção e Criação de Phytoseiulus} macropilis. Os predadores foram adquiridos da empresa PROMIP, Piracicaba, SP. A criação foi realizada em arenas, mantidas em estufa incubadora tipo BOD, à temperatura de $25 \pm 1{ }^{\circ} \mathrm{C}$, umidade relativa de $70 \pm 10 \%$ e fotofase de $12 \mathrm{~h}$. As arenas foram confeccionadas com folhas de feijão-deporco infestadas com T. urticae, sobrepostas sobre papel de filtro umedecido por uma esponja saturada em água; as folhas foram circundadas por algodão hidrófilo para evitar a fuga dos ácaros e mantidas no interior de bandejas plásticas.

Acaricidas Testados. Foram testados os acaricidas em formulação comercial: espiromesifeno $\left(\right.$ Oberon $^{\circledR} 240$ SC) e Azadiractina A/B $\left(\right.$ Azamax $^{\circledR}$ 12 CE - DVA Especialidades); e os naturais: óleos emulsionáveis de nim, Azadirachta indica A. Juss (Azadiractina 1\% - Quinabra), pinhão-manso (Jatropha curcas L.) e mamona (Ricinus communis L). Os dois últimos foram obtidos do Instituto Fazenda Tamanduá, Patos - PB, e estão em fase de desenvolvimento e experimentação.

\section{Extração dos Óleos de J. curcas e R. communis.} Frutos de J. curcas e R. communis foram coletados no campo experimental do Instituto Fazenda Tamanduá, e suas sementes prensadas a frio, separadamente, em uma prensa mecânica modelo MPE-40 da marca ECIRTEC, para obtenção dos óleos. Os óleos obtidos permaneceram cinco dias em repouso para decantação das partículas sólidas. Em seguida realizou-se a filtragem dos mesmos em papel de filtro tipo para café (Melita). Logo após, foram separados $1 \mathrm{~L}$ de cada óleo filtrado e acrescentadas $200 \mathrm{~g}$ do emulsificante Emustab e os mesmos foram misturados em liquidificador por um minuto. Nesta mistura adicionou-se 1,5 L de água e o produto foi misturado novamente em liquidificador por cinco minutos, obtendo assim os óleos emulsionáveis prontos para o uso.

Toxicidade de Acaricidas para Fêmeas Adultas de Tetranychus urticae. Discos de folhas de algodoeiro $(3,5 \mathrm{~cm} \varnothing)$ da cultivar BRS $8 \mathrm{H}$, limpos com água corrente, foram imersos nas caldas do acaricida sintético espiromesifeno, dos produtos naturais e em água destilada (testemunha), sob leve agitação, durante cinco segundos, e após 30 minutos de secagem, infestados com 15 fêmeas adultas de T. urticae retiradas da criação. Os discos foram mantidos em estufa incubadora à temperatura de 25 $\pm 1{ }^{\circ} \mathrm{C}, 70 \pm 10 \%$ de umidade relativa e fotofase de $12 \mathrm{~h}$. A mortalidade foi avaliada $48 \mathrm{~h}$ após a infestação, sendo considerados mortos os ácaros que não se moviam, vigorosamente, após um leve 
toque com pincel de pêlo fino.

Experimentos preliminares foram realizados para se estabelecer as concentrações letais $\left(\mathrm{CL}_{\mathrm{S}}\right)$ de cada produto testado. Foram estimadas as concentrações que provocassem mortalidade igual ou superior a $95 \%$ dos adultos e ovos, e as concentrações em que a mortalidade fosse semelhante à testemunha. Com base nestes dados foram estabelecidas as concentrações testadas para cada produto, tanto para adultos quanto para ovos, através da fórmula (FINNEY, 1971):

$$
q=\sqrt[n+1]{\mathrm{an}_{\mathrm{n}} / \mathrm{a}_{1}}
$$

Onde: q = razão da progressão geométrica (p.g.); $\mathrm{n}$ = número de concentrações a extrapolar; an = limite superior da p.g. (concentração que provocou mortalidade de cerca de $95 \%$, determinada por meio de teste preliminar); a1 = limite inferior da p.g. (concentração que provocou mortalidade de cerca de $5 \%$, determinada por meio de teste preliminar). Assim, foram determinadas as concentrações a serem testadas $\left(a_{1} ; a_{1} \cdot q ; a_{1} \cdot q^{2} ; a_{1} \cdot q^{3} ; a_{1} \cdot q^{4}\right.$ e $\left.a_{1} \cdot q^{5}\right)$.

Experimentos individuais foram conduzidos tendo como tratamentos cinco ou seis concentrações de cada acaricida com quatro repetições por concentração composta de 15 ácaros e tratamento testemunha sem acaricidas. Foram calculadas concentrações letais $\left(\mathrm{CL}_{50}\right.$ e $\left.\mathrm{CL}_{90}\right)$ para cada acaricida, empregando o programa POLO-PC (LEORA SOFTWARE, 1987). As Razões de Toxidade (RT) foram calculadas, através da seguinte fórmula: $\mathrm{RT}=$ maior $\mathrm{CL}_{50}$ e/ou $\mathrm{CL}_{90}$ dos acaricidas / menor $\mathrm{CL}_{50}$ e/ou $\mathrm{CL}_{90}$ dos demais, individualmente.

Toxicidade de Acaricidas para Ovos de Tetranychus urticae. Discos de folhas de algodão foram infestados com 12 fêmeas adultas de $T$. urticae durante $24 \mathrm{~h}$ para a obtenção de ovos. Em seguida, o número de ovos foi padronizado para 30 por disco foliar, os quais foram imersos nas caldas dos acaricidas em diferentes concentrações. $\mathrm{Na}$ testemunha, os discos foram imersos em água destilada. Os discos após tratamento foram deixados à temperatura ambiente por 30 minutos. Então, foram dispostos sobre papel de filtro, sobrepostos numa esponja saturada em água, no interior de bandejas plásticas, e mantidos em estufa incubadora, à temperatura de $25 \pm 1{ }^{\circ} \mathrm{C}, 70$ $\pm 10 \%$ de umidade relativa e fotofase de $12 \mathrm{~h}$. A taxa de eclosão foi avaliada 96h após a aplicação dos acaricidas, mediante contagem do número de larvas eclodidas. Os experimentos individuais foram efetuados no delineamento experimental inteiramente casualizado, tendo como tratamento cinco ou seis concentrações de cada acaricida, em quatro repetições mais tratamento testemunha. As concentrações letais $\left(\mathrm{CL}_{50}\right.$ e $\left.\mathrm{CL}_{90}\right)$ para o ácaro de cada acaricida foram calculadas através do programa POLO-PC (LEORA SOFTWARE, 1987). Com base nas CL foram calculadas as Razões de Toxidade (CL menos tóxico/CL mais tóxico).

\section{Toxicidade e Efeitos Adverso e/ou Total} de Acaricidas sobre Fêmeas de Phytoseiulus macropilis. A toxicidade e o efeito adverso e/ou total dos acaricidas sobre P. macropilis foi determinada empregando-se o método recomendado como padrão para testes em laboratório para estudos de efeito residual adaptado de Hassan et al. (1994). As arenas de 5,0 $\mathrm{cm} \varnothing$ foram semelhantes às dos experimentos anteriores, porém os discos foram circundados por algodão hidrófilo para evitar a fuga dos ácaros. Em cada disco foram colocadas 50 fêmeas adultas de $T$. urticae durante $24 \mathrm{~h}$, para efetuarem a oviposição, permanecendo, além dos ácaros, ovos (em torno de 300 ), teias e excrementos. Os discos foram imersos nas caldas de cada acaricida e na testemunha (água destilada), nas concentrações correspondentes à $\mathrm{CL}_{50}$ estimadas para T. urticae. Após a secagem, durante 30 minutos à temperatura ambiente, cada disco foi infestado com três fêmeas adultas do predador. Após o primeiro dia, diariamente foram colocadas 10 fêmeas adultas de $T$. urticae nas arenas, como alimento. Os experimentos foram realizados no delineamento inteiramente casualizado com seis tratamentos (cinco acaricidas e testemunha) empregando 10 repetições com três fêmeas do ácaro 
predador. A mortalidade das fêmeas de P. macropilis foi avaliada durante os oito dias subsequentes. Os resultados foram submetidos à análise de variância (ANOVA) e as médias comparadas pelo teste de Tukey $(\mathrm{P}=0,05)$, através do software SAS (SAS INSTITUTE, 2001).

$\mathrm{O}$ efeito adverso ou total (E\%) dos acaricidas foi calculado, levando em conta a mortalidade (corrigidas em função da mortalidade na testemunha) (ABBOTT, 1925) e o efeito na reprodução (OVERMEER; VAN ZON, 1982), através da fórmula $\mathrm{E}=100 \%-(100 \%-\mathrm{Mc}) \times \mathrm{Er}$, onde $\mathrm{Mc}=$ mortalidade corrigida e $\mathrm{Er}=$ efeito na reprodução. O efeito na reprodução (Er) foi obtido pela divisão da produção média de ovos viáveis nos tratamentos pela produção média de ovos viáveis na testemunha $(\mathrm{Er}=$ Rtratamento /Rtestemunha). A produção média de ovos $(\mathrm{R})$ foi obtida através da relação: $\mathrm{R}$ = número de ovos viáveis /número de fêmeas vivas. Durante oito dias, foram registrados diariamente, $o$ número de fêmeas vivas, bem como o número de ovos viáveis. Os efeitos totais, encontrados para cada acaricida foram classificados nas classes de 1 a 4, conforme critérios estabelecidos pela IOBC /WPRS para enquadrar produtos fitossanitários, quanto ao efeito adverso causado a organismos benéficos em testes de laboratório (BAKKER et al., 1992; HASSAN et al., 1994), sendo: classe $1=\mathrm{E}$ $<30 \%$ (inócuo ou não nocivo); classe $2=30 \%<\mathrm{E}$ $<79 \%$ (levemente nocivo); classe $3=80 \%<\mathrm{E}<$ 99\% (moderadamente nocivo) e classe $4=\mathrm{E}>99 \%$ (nocivo).

\section{Resultados e Discussão}

Toxicidade de Acaricidas para Fêmeas Adultas de Tetranychus urticae. As CLs dos acaricidas decresceram na seguinte ordem de toxicidade: espiromesifeno $>$ Azadiractina $1 \%>$ Azadiractina A/B $>R$. communis $>J$. curcas. Por outro lado, as razões de toxicidade variaram entre 12,73 e 1,05, respectivamente, para espiromesifeno e $R$. communis, em comparação a $J$. curcas. Em relação às $\mathrm{CL}_{90 \mathrm{~s}}$, espiromesifeno foi o mais tóxico e $R$. comunis o menos tóxico. As razões de toxicidade variaram de 2,43 (espiromesifeno) a 2,16 (azadiractina A/B), em relação a $R$. communis (Tabela 1).

$\mathrm{O}$ acaricida espiromesifeno foi mais tóxico que os produtos naturais, porém fica evidenciada a alta toxicidade dos produtos naturais testados, pois as $\mathrm{CL}_{50} \mathrm{~s}$ e $\mathrm{CL}_{90} \mathrm{~s}$ foram menores que a concentração comercial recomendada $(1 \%$ ou $10 \mathrm{~mL} / \mathrm{L})$. Esse desempenho é importante para o manejo integrado de ácaros fitófagos, tendo em vista as diversas vantagens dos produtos naturais em relação aos sintéticos. Estudos com outros produtos naturais como as quinonas plubagim, dibromothymoquinone e juglone sobre T. urticae resultaram em $\mathrm{CL}_{50} \mathrm{~s}$ de $0,001,0,008$ e $0,011 \%\left(\mu \mathrm{L} / \mathrm{cm}^{2}\right)$, respectivamente, demonstrando alta toxicidade (AKHTAR et al., 2012). Attia et al. (2012) estimaram $\mathrm{CL}_{50}$ de 7,49 $\mathrm{mg} / \mathrm{L}$. para o extrato de Allium sativum L. em $T$. urticae. Extratos de folhas de Aloe vera L. diluídos em acetona e etanol foram testados sobre fêmeas adultas de Tetranychus cinnabarinus (Boisduval) (Acari: Tetranychidae), apresentando $\mathrm{CL}_{50} \mathrm{~S}$ de 614 e 2235 ppm, respectivamente, após 48h de exposição, evidenciando que quando diluídos em acetona, o efeito acaricida foi potencializado (WEI et al., 2011). Estes resultados corroboram com o do presente trabalho e mostram o potencial dos produtos naturais para o manejo de T. urticae, principalmente Azadiractina A/B, R. communis e $J$. curcas que ainda são pouco estudados sobre esta praga no agroecossistema algodoeiro. 
Tabela 1. Toxicidade de diferentes acaricidas para fêmeas adultas e ovos de Tetranychus urticae, avaliada com 48 e 96 h após a aplicação.

\begin{tabular}{|c|c|c|c|c|c|c|c|c|}
\hline Acaricida & $\mathbf{n}^{1}$ & $\begin{array}{l}C_{50}(\mathrm{~mL} / \mathrm{L}) \\
\text { (I.C. a } 95 \%)\end{array}$ & $\begin{array}{l}\mathrm{CL}_{90}(\mathrm{~mL} / \mathrm{L}) \\
\text { (I.C. a } 95 \%)\end{array}$ & $\begin{array}{c}\text { Coeficiente } \\
\text { angular } \pm \\
\text { EP }\end{array}$ & $\chi^{2}$ & G.L. & $\mathrm{RT}_{50}^{2}$ & $\mathrm{RT}_{90}$ \\
\hline \multicolumn{9}{|c|}{ Fêmeas adultas - $48 \mathrm{~h}$} \\
\hline Azadiractina A/B & 300 & $\begin{array}{c}0,19 \\
(0,14-0,25)\end{array}$ & $\begin{array}{c}2,11 \\
(1,46-3,51)\end{array}$ & $1,23 \pm 0,11$ & 0,75 & 3 & 1,85 & 2,16 \\
\hline Azadiractina $1 \%$ & 300 & $\begin{array}{c}0,14 \\
(0,10-0,21)\end{array}$ & $\begin{array}{c}3,11 \\
(1,75-6,60)\end{array}$ & $0,95 \pm 0,88$ & 1,31 & 3 & 2,51 & 1,46 \\
\hline Jatropha curcas & 360 & $\begin{array}{c}0,35 \\
(0,29-0,43)\end{array}$ & $\begin{array}{c}2,09 \\
(1,56-3,01)\end{array}$ & $1,66 \pm 0,14$ & 3,90 & 4 & - & 1,66 \\
\hline Espiromesifeno & 300 & $\begin{array}{c}0,03 \\
(0,02-0,05)\end{array}$ & $\begin{array}{c}1,87 \\
(0,83-5,62)\end{array}$ & $0,70 \pm 0,072$ & 2,54 & 3 & 12,73 & 2,43 \\
\hline Ricimus communs & 360 & $\begin{array}{c}0,34 \\
(0,26-0,44)\end{array}$ & $\begin{array}{c}4,55 \\
(3,04-7,62)\end{array}$ & $1,13 \pm 0,10$ & 3,18 & 4 & 1,05 & - \\
\hline \multicolumn{9}{|l|}{ Ovos $-96 h$} \\
\hline Azadiractina A/B & 720 & $\begin{array}{c}0,26 \\
(0,21-0,33)\end{array}$ & $\begin{array}{c}4,13 \\
(2,77-6,78)\end{array}$ & $1,07 \pm 0,07$ & 2,96 & 4 & - & 1,55 \\
\hline Azadiractina $1 \%$ & 720 & $\begin{array}{c}0,17 \\
(0,13-0,23)\end{array}$ & $\begin{array}{c}4,10 \\
(2,61-7,12)\end{array}$ & $0,93 \pm 0,06$ & 3,60 & 4 & 1,53 & 1,56 \\
\hline Jatropha curcas & 720 & $\begin{array}{c}0,07 \\
(0,06-0,09)\end{array}$ & $\begin{array}{c}0,80 \\
(0,58-1,20)\end{array}$ & $1,25 \pm 0,08$ & 3,82 & 4 & 3,71 & 8,01 \\
\hline Ricimus communs & 720 & $\begin{array}{c}0,20 \\
(0,15-0,27)\end{array}$ & $\begin{array}{c}6,41 \\
(3,99-11,55)\end{array}$ & $0,86 \pm 0,06$ & 3,67 & 4 & 1,3 & - \\
\hline
\end{tabular}

${ }^{1}$ Número de ácaros/ovos utilizados em cada experimento.

${ }^{2}$ Razão de toxicidade $(\mathrm{RT})=$ maior $\mathrm{CL}_{50} \mathrm{e}_{90}$ dos acaricidas / menor $\mathrm{CL}_{50}$ e/ou $\mathrm{CL}_{90}$ dos demais para cada experimento (adultos e ovos), separadamente.

Fonte: Elaboração dos autores.

Os acaricidas sintéticos, quando utilizados de forma correta, trazem contribuição para o controle de ácaros-praga. Neste sentido, Kady et al. (2007) demonstraram que a concentração letal capaz de matar 50\% da população de fêmeas de T. urticae em discos de folha de amora (Morus Alba L.), foi 8,68 ppm para espinosina (Spinetoram 12\%) e 5,95 ppm para abamectina $\left(\right.$ Vertimec $\left.^{\circledR} 1,8 \%\right)$. Espirodiclofeno apresentou $\mathrm{CL}_{50}$ de $0,20 \mathrm{mg} \mathrm{L}^{-1}$ para a população susceptível desta praga em feijão (Phaseolus vulgaris L.) (RAUCH; NAUEN, 2003). No presente trabalho, espiromesifeno também foi muito tóxico para fêmeas de $T$. urticae em uma concentração muito baixa, mostrando que se este acaricida for utilizado de forma correta, é de extrema eficiência no controle e manejo desta praga.

Toxicidade de Acaricidas para Ovos de Tetranychus urticae. As $\mathrm{CL}_{50} \mathrm{~s}$ dos acaricidas naturais decresceram na seguinte ordem de toxicidade: $J$. curcas $>$ azadiractina $1 \%>R$. communis $>$ Azadiractina $\mathrm{A} / \mathrm{B}$, e as razões de toxicidade variaram entre 3,71 (J. curcas) e 1,3 (R. comunis). Quando se comparou as $\mathrm{CL}_{90} \mathrm{~s}$, a toxicidade dos acaricidas decresceu na seguinte ordem: $J$. curcas $>$ Azadiractina $1 \%>$ Azadiractina $\mathrm{A} / \mathrm{B}>R$. communis. Por outro lado, as razões de toxicidade apresentaram valores entre 8,01 (J. curcas) e 1,55 (Azadiractina A/B), em relação ao 
óleo de R. communis (Tabela 1).

Não foi possível calcular as $\mathrm{CL}_{50} \mathrm{~s}$ e $\mathrm{CL}_{90} \mathrm{~s}$ para espiromesifeno, pois mesmo quando testado em concentrações do produto comercial, muito baixas $(0,000015 \mathrm{~mL} / \mathrm{L})$ provocou mortalidade acima de 90\% de ovos de T. urticae, impossibilitando assim o estabelecimento da curva concentração/resposta, pois apresentou uma alta eficiência no controle de ovos desta praga. Este fato já era esperado, pois espiromesifeno é mais eficiente sobre ovos e formas imaturas do que para adultos de acordo com Nauen, Schnorbach e Elbert(2005)e Sato etal.(2011).Ainda, segundo Sato et al. (2011), ovos com idade inferior a $72 \mathrm{~h}$ são mais suscetíveis ao espiromesifeno. No presente trabalho foram utilizados ovos com idade entre 0 e $24 \mathrm{~h}$, o que pode justificar a alta eficácia de espiromesifeno sobre os mesmos. Segundo Marcic et al. (2009), espiromesifeno nas concentrações de $180 \mathrm{mg} / \mathrm{L}$ e $18 \mathrm{mg} / \mathrm{L}$, também, reduziram em 100\% a fecundidade de T. urticae, após quatro dias da aplicação, e a $1,8 \mathrm{mg} / \mathrm{L}$, a fecundidade foi reduzida em $53 \%$, em relação à testemunha.

Os acaricidas tetradifon e clorfenapir causaram $100 \%$ de mortalidade de ovos de T. urticae em mamoeiro, utilizando-se 25, 50 e 75\% da concentração comercial recomendada (ESTEVES FILHO; OLIVEIRA; GONDIN JUNIOR, 2008). Segundo os mesmos autores, a propriedade ovicida de um acaricida é relevante para a utilização em programas de manejo de ácaros-praga, pois controlando o estágio inicial de desenvolvimento da praga consequentemente reduz as injúrias e os danos causados às plantas. Resultado semelhante foi conseguido por Albuquerque et al. (2003) para o acaricida dicofol. De acordo com Duso et al. (2008), os produtos naturais azadiractina e rotenona proporcionaram 99,3 e $62,9 \%$ de inviabilidade de ovos de T. urticae, respectivamente, em feijão, nas concentrações comerciais de 4,5 g de i.a e $18 \mathrm{ml}$ de i.a/ha, recomendadas. Natuneem a $1 \%$ reduziu em 97,5\% a viabilidade de ovos de T. urticae em C. ensiformes (BRITO et al., 2006). Segundo Attia et al. (2012), o extrato de A. sativum nas concentrações 0,36 e $0,74 \mathrm{mg} / \mathrm{L}$, também foi efetivo na redução da fecundidade de $T$. urticae. No presente estudo evidenciou-se que os produtos naturais e espiromesifeno, usados em concentrações bem abaixo das recomendadas, foram eficientes no controle de ovos de T. urticae. Observou-se, também, que Azadiractina A/B e Azadiractina 1\% foram mais tóxicos para fêmeas do que para ovos de T. urticae, enquanto constatou-se o contrário para os óleos de $J$. curcas e R. communis.

Toxicidade e Efeitos Adverso e/ou Total de Acaricidas sobre Fêmeas de Phytoseiulus macropilis. A mortalidade de P. macropilis, em relação à espiromesifeno e os produtos naturais, variou entre 53,6 (espiromesifeno) a $89,3 \%$ (azadrictina $\mathrm{A} / \mathrm{B}$ ). O sintético espiromesifeno foi o que mais afetou a reprodução do predador (Er $=0,01)$, seguido pelos óleos de $J$. curcas $(0,32)$ e Azadiractina 1\% (0,74). Espiromesifeno, J. curcas e Azadiractina 1\% causaram maior efeito adverso ao predador. $\mathrm{O}$ espiromesifeno foi o único acaricida classificado na classe de toxicidade 4 e, portanto, sendo considerado nocivo ao ácaro predador (Tabela 2). Por outro lado, se consideramos apenas o resultado de mortalidade, o espiromesifeno pode ser classificado como o menos tóxico ao predador. Ao analisar os resultados de mortalidade e o efeito sobre a reprodução, o espiromesifeno comportou-se como o mais nocivo, causando um efeito adverso de 99,7\%; assim pode ser considerado incompatível para programas de manejo de T. urticae, utilizandose a associação do controle químico e biológico. 
Tabela 2. Toxicidade de diferentes acaricidas para fêmeas adultas de Phytoseiulus macropilis, em teste de contato e residual de laboratório, 8 dias após aplicação, em discos de folha de algodoeiro.

\begin{tabular}{lcccc}
\hline Acaricida & $\mathrm{Mc}^{1}$ & $\mathrm{Er}^{2}$ & $\mathrm{E}(\%)^{3}$ & Classe $^{4}$ \\
\hline Jatropha curcas & $92,8 \mathrm{a}$ & 0,32 & 97,7 & 3 \\
Azadiractina A/B & $89,3 \mathrm{a}$ & 1,61 & 79,3 & 2 \\
Ricinus communis & $85,7 \mathrm{a}$ & 1,93 & 72,5 & 2 \\
Azadiractina 1\% & $82,1 \mathrm{ab}$ & 0,74 & 86,8 & 3 \\
Espiromesifeno & $53,6 \mathrm{~b}$ & 0,01 & 99,7 & 4 \\
\hline
\end{tabular}

${ }^{1}$ Mortalidade corrigida (ABBOTT, 1925). Médias seguidas de mesma letra na coluna não diferem entre si pelo teste de Tukey (P $=0,05)$.

${ }^{2}$ Efeito sobre a reprodução. $\mathrm{E}_{\mathrm{r}}=\mathrm{R}_{\text {Tratamento }} / \mathrm{R}_{\text {Testemunha }}$

${ }^{3}$ Efeito adverso ou total. $\mathrm{E} \%=100 \%-\left(100 \%-\mathrm{M}_{\mathrm{c}}\right) \times \mathrm{E}_{\mathrm{r}}$

${ }^{4}$ Classe de toxicidade segundo IOBC/WPRS: classe $1=\mathrm{E}<30 \%$ (inócuo ou não nocivo); classe $2=30 \%<\mathrm{E}<79 \%$ (levemente nocivo); classe $3=80 \%<\mathrm{E}<99 \%$ (moderadamente nocivo) e classe $4=\mathrm{E}>99 \%$ (nocivo).

Fonte: Elaboração dos autores.

Poletti, Collette e Omoto (2008) observaram que os fungicidas, azoxistrobina, boscalida+cresoximmetílico, tebuconazol, imibenconazol, iprodiona e triforina foram inócuos a P. macropilis. Abamectina foi levemente nocivo a I. zuluagai em citros, enquanto clorfenapir, óleo vegetal e óleo mineral foram classificados como nocivos (REIS et al., 1998). Clorfenapir nas concentrações de 31,3 e 62,5 $\mathrm{mL} / 100 \mathrm{~L}$, também, foi enquadrado como nocivo ao predador Euseius alatus DeLeon, em citros (REIS; SOUSA, 2001). Entretanto, no presente trabalho, espiromesifeno foi nocivo à $P$. macropilis, enquanto que os óleos de Azadiractina A/B e $R$. communis foram levemente nocivos, indicando que esses produtos naturais são mais apropriados para o manejo de T. urticae em algodoeiro, pois afetam negativamente a população da praga e são levemente nocivos ao seu principal inimigo natural, podendo ser recomendados em programas de manejo integrado do ácaro rajado. Estes resultados em relação ao espiromesifeno corroboram com os obtidos por Irigaray e Zalom (2006) para Galendromus occidentalis (Nesbitt) (Acari: Phytoseiidae), com efeito negativo para a longevidade, a fecundidade e a fertilidade. Entretanto, Cloyd, Galle e Keith (2006) verificaram que clorfenapir e espiromesifeno nas concentrações de $0,20 \mathrm{~mL} / \mathrm{L}$ e $0,075 \mathrm{~mL} / \mathrm{L}$ em discos de folha de soja foram compatíveis com $N$. californicus, pois a população de adultos apresentou
89 e $100 \%$ de sobrevivência, respectivamente, fato corroborado por Sato et al. (2011), observando que espiromesifeno na concentração de 288 $\mathrm{mg} / \mathrm{L}$, não causou efeito negativo na ovoposição e na mortalidade de N. californicus. Contudo, clorfenapir e espiromesifeno não foram compatíveis com $P$. persimilis nas mesmas concentrações testadas para $N$. californicus, pois a população de adultos apresentou sobrevivência de 47 e 44\% respectivamente (CLOYD; GALLE; KEITH, 2006). Os mesmos autores explicaram que esta diferença pode ser devido a características fisiológicas e/ou do comportamento de forrageamento de cada predador.

Um dos maiores problemas relacionados ao controle químico de $T$. urticae é o seu elevado potencial reprodutivo e ciclo de vida curto, que favorecem o rápido desenvolvimento de resistência a acaricidas (STUMPF; NAUEN, 2001). Populações desta praga desenvolveram resistência a clorfenapir, abamectina e fenpiroximate (SATO et al., 2004), bem como ao dicofol (KIM et al., 2004, 2007; AY; KARA, 2011). Assim, são necessárias pesquisas com acaricidas de várias classes e com modos de ação diferentes, contribuindo para retardar o desenvolvimento de resistência. Neste sentido, produtos naturais vêm sendo testados, como alternativa aos acaricidas sintéticos para o controle de pragas, por sua menor possibilidade 
de desenvolver resistência, causar problemas ao ambiente ou à saúde humana (ISMAN, 2006). Isso se deve a sua bioatividade, vários modos de ação, e por provocarem efeitos repelente e antialimentar (MENEZES, 2005; ISMAN; MIRESMAILLI; MACHIAL, 2011). Outra tentativa para retardar a evolução da resistência é a associação do controle químico com outras táticas, como o controle biológico (GENTZ; MURDOCH; KING, 2010), reduzindo assim os abusos no uso de inseticidas/acaricidas, bem como contribuir para a implementação de programas de manejo integrado de T. urticae em algodoeiro.

Neste trabalho utilizou-se o inseticida/acaricida espiromesifeno, que age na biosíntese de lipídeos (IRAC, 2011), Azadiractina A/B que atua através de inibição de alimentação e crescimento, além dos produtos naturais $R$. communis e $J$. curcas, que ainda se desconhecem os seus modos de ação, sobre uma população de laboratório de T. urticae, considerada padrão de suscetibilidade. Todos os acaricidas testados foram considerados eficientes no controle de ovos e fêmeas adultas de T. urticae, demonstrando que se este ácaro for manejado de forma adequada, dentro dos conceitos do MIP, fazendo-se rotação de produtos com modos de ação distintos, diminuiria a chance do mesmo desenvolver resistência. Entretanto, $R$. communis e Azadiractina A/B foram considerados levemente nocivos para P. macropilis, sendo assim, os mais promissores para o manejo de T. urticae no agroecossistema algodoeiro.

Com base nos resultados obtidos no presente trabalho, futuras pesquisas devem ser incentivadas no sentido de se avaliar a interação dos acaricidas botânicos, espiromesifeno e P. macropilis, em casade-vegetação e campo, visando ao manejo de $T$. urticae em algodoeiro.

\section{Agradecimentos}

À CAPES/FACEPE e ao CNPq, pela concessão de bolsas de estudo e de produtividade em pesquisa. À PROMIP, pelo envio dos ácaros predadores.

\section{Referências}

ABBOTT, W. S. A method of computing the effectiveness of an insecticide. Journal of Economic Entomology, Lanham, v. 18, n. 1, p. 265-267, 1925.

AGROFIT - Sistema de Agrotóxicos Fitossanitários. 2011. Disponível em: <http://extranet.agricultura.gov. br/agrofit_cons/principal_agrofit_cons $>$. Acesso em: 10 dez. 2011.

AKHTAR, Y.; ISMAN, M. B.; LEE, C. H.; LEE, S. H.; LEE, H. S. Toxicity of quinones against two-spotted spider mite and three species of aphids in laboratory and greenhouse conditions. Industrial Crops and Products, San Diego, v. 37, n. 1, p. 536-541, 2012.

ALBUQUERQUE, F. A.; OLIVEIRA, J. V.; GONDIM JUNIOR, M. G. C.; TORRES, J. B. Efeito de inseticidas e acaricidas sobre ovos e fêmeas adultas do ácaro rajado, Tetranychus urticae Koch (Acari: Tetranychidae). Pesticidas: Revista de Ecotoxicologia e Meio Ambiente, Curitiba, v. 13, p. 1-8, 2003.

ASHLEY, J. L.; HERBERT, D. A.; LEWIS, E. E.; BREWSTER, C. C.; HUCKABA, R. Toxicity of three acaricides to Tetranychus urticae (Tetranychidae: Acari) and Orius insidiosus (Anthocoridae: Hemiptera). Journal of Economic Entomology, Lanham, v. 99, n. 1, p. 54-59, 2006.

ATTIA, S.; GRISSA, K. L.; MAILLEUX, A. C.; LOGNAY, G.; HEUSKIN, S.; MAYOUFI, S.; HANCE, T. Effective concentrations of garlic distillate (Allium sativum) for the control of Tetranychus urticae (Tetranychidae). Journal of Applied Entomology, Berlin, v. 36, n. 4, p. 302-312, 2012.

AY, R.; KARA, F. E. Toxicity, inheritance of fenpyroximate resistance, and detoxification-enzyme levels in a laboratory-selected fenpyroximate-resistant strain of Tetranychus urticae Koch (Acari: Tetranychidae). Crop Protection, Guildford, v. 30, n. 6, p. 605-610, 2011.

BAKKER, F.; GROVE, A.; BLUMEL, S.; CALIS, J.; OOMEN, P. Side-effect tests for phytoseiids and their rearing methods. IOBC/WPRS Bull, Darmstadt, v. 15, n. 3, p. 61-81, 1992.

BRITO, H. M.; GONDIM JUNIOR, M. G. C.; OLIVEIRA, J. V.; CÂMARA, C. A. G. Toxicidade de Natuneen sobre Tetranychus urticae Koch (Acari: Tetranychidae) e ácaros predadores da família Phytoseiidae. Ciência e Agrotecnologia, Lavras, v. 30, n. 4, p. 685-691, 2006.

CASTAGNOLI, M.; ANGELI, G.; LIGUORI, M.; FORTI, D.; SIMONI, S. Side effects of botanical insecticides on predatory mite Amblyseius andersoni (Chant). Journal of Pest Science, Heidelberg, v. 75, n. 5, 
p. $122-127,2002$.

CLOYD, R. A.; GALLE, C. L.; KEITH, S. R. Compatibility of three miticides with the predatory mites Neoseiulus californicus McGregor and Phytoseiulus persimilis Athias-Henriot (Acari: Phytoseiidae). Hortscience, Alexandria, v. 44, n. 3, p. 476-480, 2006.

DEGRANDE, P. E.; REIS, P. R.; CARVALHO, G. A.; BELARMINO, L. C. Metodologia para avaliar o impacto de pesticidas sobre inimigos naturais,. In: PARRA, J. R. P.; BOTELHO, P. S. M.; CORRÊA-FERREIRA, B. S.; BENTO, J. M. S. (Ed.). Controle biológico no Brasil: parasitóides e predadores. São Paulo, Manole, 2002. p. 71-93.

DUSO, C.; MALAGNINI, V.; POZZEBON, A.; CASTAGNOLI, M.; LIGUORI, M.; SIMONI, S. Comparative toxicity of botanical and reduced-risk inseticides to Mediterranean populations of Tetranychus urticae and Phytoseiulus persimilis (Acari Tetranychidae, Phytoseiidae). Biological Control, Orlando, v. 47, n. 1, p. 16-21, 2008.

ESTEVES FILHO, A. B.; OLIVEIRA, J. V.; GONDIM JUNIOR, M. G. C. Toxicidade de acaricidas sobre diferentes estágios de vida de Tetranychus urticae Koch (Acari: Tetranychidae) em Mamoeiro. BioAssay, Piracicaba, v. 3, n. 6, p. 1-6, 2008.

ESTEVES FILHO, A. B.; OLIVEIRA, J. V.; TORRES, J. B.; GONDIM JUNIOR, M. G. C. Biologia comparada e comportamento de Tetranychus urticae Koch (Acari: Tetranychidae) e Phytoseiulus macropilis (Banks) (Acari: Phytoseiidae) em algodoeiro Bollgard ${ }^{\mathrm{TM}}$ e Isolinha nãotransgênica. Neotropical Entomolology, Londrina, v. 39, n. 4, p. 338-344, 2010.

FERLA, N. J.; MARCHETTI, M. M.; GONÇALVES, D. Ácaros predadores (Acari) associados à cultura do morango (Fragaria sp, Rosaceae) e plantas próximas no Estado do Rio Grande do Sul. Biota Neotropica, Campinas, v. 7, n. 2, p. 1-8, 2007.

FERLA, N. J.; MARCHETTI, M.; JOHANN, L.; HAETINGER, C. Functional response of Phytoseiulus macropilis under different Tetranychus urticae (Acari: Phytoseiidae, Tetranychidae) population density in laboratory. Zoologia, Curitiba, v. 28, n. 1, p. 17-22, 2011.

FINNEY, D. J. Probit analysis. 3. ed. London: Cambridge Press, $1971.338 \mathrm{p}$.

GENTZ, M. C.; MURDOCH, G.; KING, G. F. Tandem use of selective insecticides and natural enemies for effective, reduced risk pest management. Biological Control, Orlando, v. 52, n. 3, p. 208-215, 2010.

GRBIC, M.; LEEUWEN, T. V.; CLARK, R. M.; ROMBAUTS, S.; ROUZE, P.; GRBIC, V.; OSBORNE,
E. J.; DERMAUW, W.; NGOC, P. C. T.; ORTEGO, F.; HERNÁNDEZ-CRESPO, P.; DIAZ, I.; MARTINEZ, M.; NAVAJAS, M.; SUCENA, E.; MAGALHÃES, S.; NAGY, L.; PACE, R. M.; DJURANOVIC, S.; SMAGGHE, G.; IGA, M.; CHRISTIAENS, O.; VEENSTRA, J.A.; EWER, J.; VILLALOBOS, R. M.; HUTTER, J. L.; HUDSON, S. D.; VELEZ, M.; YI, S. V.; ZENG, J.; SILVA, A. P.; ROCH, F.; CAZAUX, M.; NAVARRO, M.; ZHUROV, V.; ACEVEDO, G.; BJELICA, A.; FAWCETT, J. A.; BONNET, E.; MARTENS, C.; BAELE, G.; WISSLER, L.; SANCHEZ-RODRIGUEZ, A.; TIRRY, L.; BLAIS, C.; DEMEESTERE, K.; HENZ, S. R.; GREGORY, T. R.; MATHIEU, J.; VERDON, L.; FARINELLI, L.; SCHMUTZ, J.; LINDQUIST, E.; FEYEREISEN, R.; DE PEER, Y. V. The genome of Tetranychus urticae reveals herbivorous pest adaptations. Nature, London, v. 479, n. 7374, p. 487-492, 2011.

HASSAN, S. A.; BIGLER, F.; BOGENSCHÜTZ, H.; BOLLER, E.; BRUN, J.; CALIS, J. N. M.; COREMANS-PELSENEER, J.; DUSO, D.; GROVER, A.; HEIMBACH, U.; HELYER, N.; HOKKANEN, H.; LEWIS, G. B.; MANSOUR, F.; MORETH, L.; POLGAR, L.; SAMSOE-PETERSEN, L.; SALPHANOR, B.; STÄUBLI, A.; STERK, G.; VAINIO, A.; VAN DE VEIRE, M.; VIGGIANI, G.; VOGT, H. Results of the sixth joint pesticide testing programme of the IOBC / WPRS - working group pesticides and beneficial organisms. Entomophaga, Paris, v. 39, n. 1, p. 109-119, 1994.

HOY, M. A. Agricultural acarology: introduction to integrated mite management. Boca Raton: CRC Press, $2011.410 \mathrm{p}$.

IRAC - Comitê Brasileiro de Ação a Resistência a Inseticidas. $<$ Disponível em: http://www.irac-br.org.br/>. Acesso em: 22 nov. 2011.

IRIGARAY, F. J.S. C.; ZALOM, F. G. Side effects of five new acaricides on the predator Galendromus occidentalis (Acari, Phytoseiidae). Experimental and Applied Acarology. Amsterdam, v. 38, n. 4, p. 299-305, 2006.

ISMAN, M. B. Botanical insecticides, deterrents, and repellents in modern agriculture and an increasingly regulated world. Annual Review of Entomology, Stanford, v. 51, n. 1, p. 45-66. 2006.

ISMAN, M. B.; MIRESMAILLI, S.; MACHIAL, C. Commercial opportunities for pesticides based on plant essential oils in agriculture, industry and consumer products. Phytochemistry Reviews, v. 10, n. 2, p. 197204, 2011.

KADY, G. A. E.; SHARABASY, H. M. E.; MAHMOUD, M. F.; BAHGAT, I. M. Toxicity of two potential bioinsecticides against moveable stages of Tetranychus 
urticae Koch. Journal of Applied Sciences Research, v. 3, n. 11, p. 1315-1319, 2007.

KIM, Y. J.; LEE, S. H.; LEE, S. W.; AHN, Y. J. Fenpyroximate resistance in Tetranychus urticae (Acari: Tetranychidae): cross-resistance and biochemical resistance mechanisms. Pest Management Science, Sussex, v. 60, n. 10, p. 1001-1006, 2004.

KIM, Y. J.; LEE, S. W.; CHO, J. R.; PARK, H. M.; AHN, Y. J. Multiple resistance and biochemical mechanisms of dicofol resistance in Tetranychus urticae (Acari: Tetranychidae). Journal of Asia-Pacific Entomology, San Diego, v. 10, n. 2, p. 165-170, 2007.

LeOra Software. POLO PC: probit or logit analysis [computer program]. Califórnia, USA: Berkeley Publishing House, 1987.

MARCIC, D.; OGURLIC, I.; MUTAVDZIC, S.; PERIC, $\mathrm{P}$. The Effect of spiromesifen on the reproductive potential of Tetranychus urticae koch (Acari: Tetranychidae). Pesticidi i fitomedicina, Belgrado, v. 24, n. 3, p. 203-209, 2009.

MARCIC, M. Sublethal effects of spirodiclofen on life history and life-table parameters of two-spotted spider mite (Tetranychus urticae). Experimental and Applied Acarology, Amsterdam, v. 42, n. 2, p. 121-129, 2007.

MARTINEZ-VILLAR, E.; SÁENZ-DE-CABESÓN, F. J.; MORENO-GRIJALBA, F.; MARCO, V.; PÉREZMORENO, I. Effects of azadirachtin on the two-spotted spider mite, Tetranychus urticae (Acari: Tetranychidae). Experimental and Applied Acarology, Amsterdam, v. 35, n. 3, p. 215-222, 2005.

MCMURTRY, J. A.; CROFT, B. A. Life-styles of phytoseiid mites and their roles in biological control. Annual Review of Entomology, Stanford, v. 42, p. 291321, 1997.

MENEZES, E. L.A. Inseticidas botânicos: seus princípios ativos, modo de ação e uso agrícola. Seropédica: Embrapa Agrobiologia, 2005. 58 p. (Documentos, 205).

MIRESMAILLI, S.; ISMAN, M. B. Efficacy and persistence of Rosemary oil as acaricide against twospotted spider mite (Acari: Tetranychidae) on greenhouse tomato. Journal of Economic Entomology, Lanham, v. 99, n. 6, p. 2015-2023, 2006.

MOURÃO, S. A.; SILVA, J. C. T.; GUEDES, R. N. C.; VENZON, M.; JHAM, G. N.; OLIVEIRA, C. L.; ZANUNCIO, J. C. Seletividade de extratos de nim (Azadirachta indica A. Juss.) ao ácaro predador Iphiseiodes zuluagai Denmark \& Muma (Acari: Phytoseiidae). Neotropical Entomology, Londrina, v. 33, n. 5, p. 613-617, 2004.
NAUEN, R.; SCHNORBACH, H.-J.; ELBERT, A. The biological profile of spiromesifen (Oberon $\AA$ ) - A new tetronic acid insecticide/acaricide. PflanzenschutzNachrichten Bayer, Leverkusen, v. 58, n. 76, p. 417-440, 2005.

OLIVEIRA, C. A. L.; CALCAGNOLO, G. Ação do ácaro "rajado" Tetranychus urticae (Koch, 1836) na depreciação quantitativa da produção algodoeiro. $O$ Biológico, São Paulo, v. 41, p. 307-327, 1975.

OLIVEIRA, H.; FADINI, M. A. M.; VENZON, M.; REZENDE, D.; REZENDE, F.; PALINI, A. Evaluation of the predatory mite Phytoseiulus macropilis (Acari: Phytoseiidae) as a biological control agent of the two-spotted spider mite on strawberry plants under greenhouse conditions. Experimental and Applied Acarology, Amsterdam, v. 47, n. 4, p. 275-283, 2009.

OVERMEER, W. P. J.; VAN ZON, A. Q. A standardized method for testing the side effects of pesticides on the predaceous mite, Amblyseius potentillae (Acarina: Phytoseiidae). Entomophaga, Paris, v. 27, n. 4, p. 357364, 1982.

POLETTI, M.; COLLETTE, L. P.; OMOTO, C. Compatibilidade de agrotóxicos com os ácaros predadores Neoseiulus californicus (McGregor) e Phytoseiulus macropilis (Banks) (Acari: Phytoseiidae). BioAssay, Piracicaba, v. 3, n. 3, p.1-14, 2008.

PONTES, W. J. T.; OLIVEIRA, J. C. G.; CÂMARA, C. A. G.; ASSIS, C. P. O.; OLIVEIRA, J. V.; GONDIN JÚNIOR, M. G. C.; BARROS, R. Effects of the ethanol extracts of leaves and branches from four species of the genus Croton on Tetranychus urticae Koch (Acari: Tetranychidae). BioAssay, Piracicaba, v. 6, n. 3, p. 1-5, 2011.

RAUCH, N.; NAUEN, R. Spirodiclofen resistance risk assessment in Tetranychus urticae (Acari: Tetranychidae): a biochemical approach. Pesticide Biochemistry and Physiology, San Diego, v. 74, n. 2, p. 91-101, 2003.

REIS, P. R.; CHIAVEGATO, L. G.; ALVES, E. B.; SOUSA, E. O. Ácaros da família Phytoseiidae associados aos citros no Município de Lavras, Sul de Minas Gerais. Anais da Sociedade Entomológica do Brasil, Jaboticabal, v. 29, n. 1, p. 95-104, 2000.

REIS, P. R.; CHIAVEGATO, L. G.; MORAES, G. J. Seletividade de agroquímicos ao ácaro predador Iphiseiodes zuluagai Demark \& Muma (Acari: Phytoseiidae). Anais da Sociedade Entomológica do Brasil, Jaboticabal, v. 27, n. 2, p. 265-274, 1998.

REIS, P. R.; SOUSA, E. O. Seletividade de chlorfenapyr e fenbutatin-oxide sobre duas espécies de ácaros predadores (Acari: Phytoseiidae) em citros. Revista 
Brasileira de Fruticultura, Cruz das Almas, v. 23, n. 3, p. 584-588, 2001.

SAS Institute. SAS/STAT User's guide, version 8.2, TS level 2MO. SAS Institute. Inc., Cary, N.C. 2001.

SATO, M. E.; MIYATA, T.; SILVA, M.; RAGA, A.; SOUZA FILHO, M. F. Selections for fenpyroximate resistance and susceptibility, and inheritance, crossresistance and stability of fenpyroximate resistance in Tetranychus urticae Koch (Acari: Tetranychidae). Applied Entomology and Zoology, Tokyo, v. 39, n. 2, p. 293-302, 2004.

SATO, M. E.; SILVA, M. Z.; RAGA, A.; CANGANI, K. G.; VERONEZ, B.; NICASTRO, R. L. Spiromesifen toxicity to the spider mite Tetranychus urticae and selectivity to the predator Neoseiulus californicus. Phytoparasitica, Bet Dagan, v. 39, n. 5, p. 437-445, 2011.

SATO, M. E.; SILVA, M. Z.; CANGANI, K. G.; RAGA, A. Seleções para resistência e suscetibilidade, detecção e monitoramento da resistência de Tetranychus urticae ao acaricida clorfenapir. Bragantia, Campinas, v. 66, n. 1, p. 89-95, 2007.

SATO, M. E.; SILVA, M. Z.; RAGA, A.; SOUZA FILHO, M. F. Abamectin resistence in Tetranychus urticae Koch (Acari: Tetranychidae): selection, cross-resistence and stability of resistence. Neotropical Entomolology, Londrina, v. 34, n. 6, p. 991-998, 2005.
STUMPF, N.; NAUEN, R. Cross-resistance, inheritance, and biochemistry of mitochondrial electron transport inhibitor-acaricide resistance in Tetranychus urticae (Acari: Tetranychidae). Journal of Economic Entomology, Lanham, v. 94, n. 6, p. 1577-1583, 2001.

TORRES, J. B. Controle de pragas do algodoeiro: expectativas de mudanças. Ciência Agrícola, Maceió, v. 8, n. 1, p. 37-49, 2008.

VASCONCELOS, G. J. N.; SILVA, F. R.; BARBOSA, D. G. F.; GONDIM JUNIOR, M. G. C.; MORAES, G. J. Diversidade de fitoseídeos (Acari: Phytoseiidae) em fruteiras tropicais no estado de Pernambuco, Brasil. Magistra, Cruz das Almas, v. 18, n. 2, p. 90-101, 2006.

WATANABE, M. A.; MORAES, G. J.; GASTALDO JUNIOR, I.; NICOLELLA, G. Controle biológico do ácaro rajado com ácaros predadores fitoseídeos (Acari: Tetranychidae, Phytoseiidae) em culturas de pepino e morango. Scientia Agricola, Piracicaba, v. 51, n. 1, p. $75-$ 81, 1994.

WEI, J.; DING, W.; ZHAO, Y.; VANICHPAKORN, P. Acaricidal activity of Aloe vera L. leaf extracts against Tetranychus cinnabarinus (Boisduval) (Acarina: Tetranychidae). Journal of Asia-Pacific Entomology, San Diego, v. 14, n. 3, p. 353-356, 2011. 